\title{
Title: Ligand-Metal Cooperation Enables C-C Activation Cross-Coupling Reactivity of Cyclopropyl Ketones
}

\author{
Authors: Michael M. Gilbert, Michael J. Trenerry, Victoria R. Longley, John F. Berry, Daniel J. \\ Weix $^{1 *}$

\begin{abstract}
Affiliations:
${ }^{1}$ Department of Chemistry, University of Wisconsin-Madison; Madison, WI, USA 53706

*Corresponding author. Email: dweix@wisc.edu
\end{abstract}

\begin{abstract}
Activation of $\mathrm{C}-\mathrm{C}$ bonds has the potential to revolutionize how molecules are made by altering the carbon skeleton and enabling new synthetic routes. Stereodefined cyclopropyl ketones have become readily available and would be an ideal source of linear 3-carbon fragments, but this reactivity is unknown. In this study we show how a new type of $\mathrm{C}-\mathrm{C}$ activation catalyst, that relies upon a different, metalloradical mechanism, can enable new subsequent reactivity: the crosscoupling of cyclopropyl ketones with organozinc reagents and chlorotrimethylsilane to form 1,3difunctionalized, ring-opened products. A mixture of experiment and theory sheds light on how cooperation between the redox-active ligand and the nickel catalyst enables the $\mathrm{C}-\mathrm{C}$ bond activation step, suggesting how this approach could be applied in other systems.
\end{abstract}

One-Sentence Summary: A new $\mathrm{C}-\mathrm{C}$ activation mechanism unlocks new subsequent reactivity and a new approach to using cyclopropyl ketones in synthesis. 


\section{Main text:}

The selective activation of $\mathrm{C}\left(\mathrm{sp}^{3}\right)-\mathrm{C}\left(\mathrm{sp}^{3}\right)$ bonds by transition metals has the potential to revolutionize organic synthesis, but presents a considerable fundamental challenge. Synthetically, catalytic $\mathrm{C}-\mathrm{C}$ bond cleavage coupled with the formation of new $\mathrm{C}-\mathrm{C}$ bonds could enable novel retrosynthetic strategies based on converting an accessible carbon skeleton into a more desirable scaffold. The underlying challenge in the development of $\mathrm{C}-\mathrm{C}$ activation reactions is the low reactivity of $\mathrm{C}-\mathrm{C}$ bonds compared to other functional groups, even when activated by ring strain or directing groups $(1,2)$. The focus to date, regardless of transition metal, has been on the development of electron-rich, low-coordinate metal centers with strong $\sigma$-donor ligands to overcome the low reactivity of $\mathrm{C}-\mathrm{C}$ bonds towards oxidative addition (3). This strategy has enabled the discovery of many new reactions, such as ring expansions with $\pi$ components, but broadening the reactivity of the metallacyclic intermediates derived from cyclopropane oxidative addition remains an outstanding challenge $(4,5)$. For example, while nickel-catalyzed "cut-and-sew" reactions of cyclopropyl ketones with alkenes and alkynes has transformed how cyclopentanes are synthesized (6-9), difunctionalization to acyclic products has been elusive (Fig. 1A) (10). We show here a new strategy for cyclopropane $\mathrm{C}-\mathrm{C}$ activation that enables the resulting intermediates to be connected to different organometallic steps for the formation of acyclic products (Fig. 1B). This approach relies on the use of highercoordinate, $\pi$-acceptor ligands that store significant spin-density and enable metalloradical bond activation as opposed to concerted oxidative addition. These catalysts open the strained ring system of cyclopropyl ketones by generating ketyl carbon-radical character on the substrate that leads to a concerted metalloradical $\mathrm{C}-\mathrm{C}$ bond rearrangement and rebound to form a functionalized alkylnickel intermediate suitable for cross-coupling with organozinc reagents.

This metalloradical $\mathrm{C}-\mathrm{C}$ activation/cross-coupling approach could have immediate synthetic impact if applied to the linearization of cyclopropyl ketones to versatile, substituted acyclic silyl enol ethers (Fig. 1C). The power of this approach capitalizes on the host of recent advances in cyclopropane synthesis and derivatization, that has transformed these formerly inaccessible target molecules into viable starting materials (11-16). The organized transition state of cyclopropanations, as well as the rigidity of the cyclopropane scaffold, means that many of these reactions proceed with high stereocontrol. General approaches to open these stereodefined rings into linear fragments would address challenges in acyclic stereocontrol (17). Finally, the immediate versatility of $\mathrm{C}-\mathrm{C}$ activation/1,3-difunctionalization is maximized if one site could be further diversified by a collection of reactions, such as with a silyl enol ether, allowing subsequent $\mathrm{C}-\mathrm{C}, \mathrm{C}-\mathrm{O}$, and $\mathrm{C}-\mathrm{N}$ bond forming transformations (18$3520)$.

Early in reaction development we recognized that the ligands that promote concerted nickel(0) oxidative addition of cyclopropyl ketones, such as monodentate phosphines and $N$ heterocyclic carbenes, form metallacyclic intermediates that are unreactive for Negishi crosscoupling reactions $(6-10)$. On the other hand, while it is known that bi- and tridentate $N$-donor $40 \quad$ ligands promote cross-coupling reactions with alkyl halides to form $\mathrm{C}\left(\mathrm{sp}^{2}\right)-\mathrm{C}\left(\mathrm{sp}^{3}\right)$ and $\mathrm{C}\left(\mathrm{sp}^{3}\right)-$ $\mathrm{C}\left(\mathrm{sp}^{3}\right)$ bonds (21-25), these ligands have not been reported for $\mathrm{C}-\mathrm{C}$ bond activation reactions. Building upon studies showing tridentate $N$-donor ligands can promote single electron transfer (SET)-based reactions (26), our solution to this long-standing challenge relies upon forcing a well-known cross-coupling catalyst, nickel terpyridine ((tpy)Ni), into a reactive, reduced state, that turns on $\mathrm{C}-\mathrm{C}$ activation activity while retaining cross-coupling activity (Fig. 1D). In analogy to reports with titanium, a SET mechanism from a metalloradical nickel intermediate 
to a cyclopropyl ketone accomplishes the requisite $\mathrm{C}-\mathrm{C}$ activation through a radical rearrangement (27). In contrast to other systems, the (tpy)Ni catalyst allows concomitant oxidative capture of the transient radical intermediate to generate a versatile alkylnickel(II) complex. This fast, favorable capture step allows the opening of cyclopropyl ketones to form reactive primary radicals, a challenge for other metalloradical approaches (27).

Consistent with this reasoning, preliminary studies found that (tpy) Ni complexes are effective at catalyzing the cross-coupling of ( $p$-tolyl)ZnI and chlorotrimethylsilane (TMSCl) with phenyl cyclopropyl ketone (1a) to form the acyclic silyl enol ether cross-coupled product (3a) (Fig. 2A). While catalysts derived from a variety of polypyridine ligands provided measurable product, terpyridine (tpy) was the most effective, affording 3a in $80 \%$ yield and $>95: 5 \quad Z: E$ stereoselectivity (Fig. S1). Reactions performed in the presence of a substoichiometric amount of $\mathrm{Zn}$, even for redox-neutral reactions, gave superior outcomes. We attribute these findings to the fast oxidation and deactivation of the key (tpy $\left.{ }^{\circ-}\right) \mathrm{Ni}^{\mathrm{I}}$ intermediate by other $\left[\mathrm{Ni}^{\mathrm{II}}\right]$ species in solution (vide infra).

A collection of experimental and theoretical studies (Fig. 2) allow us to propose a mechanism for this new reaction (Fig. 2A), explain the need for Zn reductant ([1] in Fig. 2), show that a $\left(\right.$ tpy $\left.^{-}\right) \mathrm{Ni}^{\mathrm{I}}$ intermediate is required ([2] in Fig. 2), and strongly support the proposed metalloradical $\mathrm{C}-\mathrm{C}$ activation step ([3] and [4] in Fig. 2). Our proposed mechanism is shown in Fig. 2A. Reduction of the (tpy)Ni(II) pre-catalyst affords neutral nickel intermediate (tpy ${ }^{*}$ ) $\mathrm{Ni}^{\mathrm{I} L}$ (I-NMP, L = NMP), which coordinates to the TMSCl-activated carbonyl (II). A single electron transfer from (tpy $\left.{ }^{\bullet-}\right) \mathrm{Ni}^{\mathrm{I}}$ to the coordinated cyclopropyl ketone, followed by concerted oxidative ring opening of the ketyl-like intermediate, affords homoallyl nickel(II) intermediate III. Transmetalation with an organozinc reagent and subsequent reductive elimination forms the cross-coupled product and turns over the catalytic cycle.

First, our experimental studies are consistent with $\mathrm{C}-\mathrm{C}$ activation by $\left(\right.$ tpy $\left.{ }^{\bullet-}\right) \mathrm{Ni}^{\mathrm{I}}(\mathbf{I})$ and allow us to rule out the intermediacy of (tpy) $\mathrm{Ni}^{\mathrm{I} X}(\mathrm{X}=\mathrm{Cl}$, I) (Fig. 2B, entries 1-5, Fig. S9). Furthermore, we observed that cyclopropane $\mathrm{C}-\mathrm{C}$ activation can be induced by (tpy) $\mathrm{Ni}^{\mathrm{I}} \mathrm{Cl}$ in the presence of $\mathrm{Zn}$, indicating that $\mathrm{Zn}$ can generate I-NMP under conditions analogous to the catalytic reaction (Fig. 2B, entry 6). Regardless of how the complexes were generated (preformed or in situ via reductants), only I-NMP reacted to form products derived from ringopening silylation (19' and 19') intermediate commonly invoked in nickel(I/III) Negishi cross-coupling reactions with organohalides, does not react with cyclopropyl ketone $\mathbf{1 a}$ to form the cross-coupled product. (Fig. 2C, Fig. S10) $(21,24)$.

The useful $\mathrm{C}-\mathrm{C}$ bond activation reactivity of this reduced (tpy)Ni catalyst is a consequence of metal-ligand cooperativity. Consistent with studies by Vicic $(21,28)$, Chirik (29), and Weighardt (30), our DFT studies show that the (tpy)Ni catalyst system exhibits redox changes at both the metal and the ligand. These calculations predict a radical anion tpy ligand and formal nickel(I) oxidation state for neutral complexes (Fig. 3A-C, Fig. S19, Fig. S26-S28) (31). In each case, a triplet ground state is favored with unpaired electrons in nickel $d_{x^{2}-y^{2}}$ and tpy $\pi^{*}$ singly-occupied molecular orbitals (SOMOs). Ferromagnetic coupling of the unpaired electrons to give an overall $S=1$ spin state is consistent with the GoodenoughKanamori rules, as the two SOMOs possess orthogonal symmetries (32).

Considering the ubiquity of (tpy)Ni complexes in cross-coupling, and reports on the high reactivity of $\mathrm{Ni}^{0}(\mathrm{cod})_{2}$ with tpy in oxidative addition (33), it is surprising that $\mathrm{C}-\mathrm{C}$ activation 
reactivity has not been reported. This is because sustained access to I-NMP in the absence of a reductant under Negishi cross-coupling conditions is not possible due to fast catalyst speciation. We confirmed that $\left(\mathrm{tpy}^{-}\right) \mathrm{Ni}^{\mathrm{II}}(p$-tolyl) is the major observable nickel species obtained from the reduction of (tpy) $\mathrm{Ni}^{\mathrm{II}} \mathrm{Cl}_{2}$ by ( $p$-tolyl)ZnI (Fig. S6-S8). DFT studies indicate that comproportionation to form (tpy) $\mathrm{Ni}^{\mathrm{I} X}$ from I-NMP and (tpy) $\mathrm{Ni}^{\mathrm{II}} \mathrm{X}_{2}$ (X = halide) is essentially irreversible ( $\mathrm{X}=\mathrm{Cl}, \Delta \mathrm{G}=-20.6 \mathrm{kcal} / \mathrm{mol})$ (Fig. S25). Furthermore, experiments and calculations demonstrate that speciation between I-NMP and $\mathbf{4}$ to form $\left(\operatorname{tpy}{ }^{\circ}\right) \mathrm{Ni}^{\mathrm{II}}(2-$ anisyl) is fast and highly favorable (Fig. S12, Fig. S24). Together, these results explain why maintaining a productive $\left(\mathrm{tpy}^{\circ}\right) \mathrm{Ni}^{\mathrm{I}}$ form of the catalyst in the absence of a reductant is challenging, even when using a nickel(0) precatalyst (as in Fig. 2D, entries 1-5). We note that in the natural world, external reductant systems are used to maintain unsustainable oxidation states in enzymatic catalysis, for example the recovery of off-cycle of cobalt(II) to cobalt(I) in methionine synthase by methionine synthase reductase (34).

Consistent with these findings, low conversion of cyclopropyl ketone 1a to product 3a was observed without an external reductant (2-6 turnovers), regardless of the oxidation state of the starting catalyst (as in Fig. 2D, entries 1-5). This suggests that, as above, the active nickel catalyst I-NMP is competitively deactivated and requires a "reductase-like" external reducing force to re-establish the reduced tpy species (Fig. 2D, entries 6-10, 12). We found that either chemical $\left(\mathrm{Zn}, \mathrm{E}_{1 / 2}=-1.38 \mathrm{~V}\right.$ vs. $\left.\mathrm{Fc} / \mathrm{Fc}^{+}\right)(35,36)$ or electrochemical $(-1.36 \mathrm{~V}$ vs. $\mathrm{Fc} / \mathrm{Fc}^{+}$) reductants enabled sustained access to I-NMP and reactions with reductants provided improved yields of 3a and increased turnover numbers (52-79\% yield, 10-16 turnovers).

DFT studies shed light on how the unusual electronic structure of $\mathbf{I}$ enables cyclopropane $\mathrm{C}-\mathrm{C}$ scission under these reaction conditions. Initial activation of the cyclopropyl ring appears dependent on the interaction of the cyclopropyl ketone substrate with both TMSCl and (tpy)Ni components, as DFT results do not predict substrate-based radical character in the electronic ground states of I-CPK $(\mathbf{L}=\mathbf{1 a})$, where the cyclopropyl ketone substrate binds to I but TMSCl is absent (Fig. 3A-C). This interpretation is consistent with experimental data: there is negligible substrate consumption by I-NMP in the absence of TMSCl, but rapid substrate consumption when TMSCl is present (Fig. 2E, entries 1,3).

The reason for this change in reactivity with $\mathrm{TMSCl}$ is that a transient silyl carboxonium ion, formed by interaction of $\mathrm{TMSCl}, \mathrm{ZnX}_{2}$, and the ketone, is a better electron acceptor that reacts with I to form a ketyl-like II (Fig. 3 A-C) that rearranges and recombines with the catalyst to form III (Fig. 3D) (37). The silyl carboxonium ion, upon binding to I, accepts electron density from the tpy ${ }^{\circ}$ ligand through an intramolecular SET event that confers significant spin density (i.e., radical character) to the $\mathrm{p}_{z}$ orbital of the carbonyl carbon to form an $O$-nickel-coordinated $\alpha$-silyloxy radical (Fig. 3A-C). While free cyclopropylmethyl radicals undergo rapid radical ring-opening processes $\left(\mathrm{k}_{\text {rearrangement }} \sim 10^{7} \mathrm{~s}^{-1}\right)(38)$, radical intermediate II instead participates in an asychronous concerted oxidative ring opening with a low barrier (Fig. 3D) to break the $\mathrm{C}-\mathrm{C}$ bond and form a new $\mathrm{Ni}-\mathrm{C}$ bond. The resulting intermediate metallacycle species III, a square-planar nickel(II) complex with a weak apical silyl-ether interaction, favors a $S=0$ spin state, indicating that the overall transformation from II to III ( $\Delta \mathrm{G}$ $=-23.2 \mathrm{kcal} / \mathrm{mol}$ ) involves intersystem crossing. Experimentally, analysis of an aliquot of the catalytic reaction of 1a and 4-iodotoluene in the presence of a spin trap was EPR silent, consistent with a diamagnetic III resting state and disfavoring any mechanisms involving longer-lived radicals (see supplementary information section V for experimental details) (27). This coordination/reduction/insertion sequence also helps explain the observed $Z$-enol-ether geometry in the product (Fig. S23). Finally, unlike $C, O$-metallacycles formed with 
monophosphine ligands, III has a weakly-bound oxygen ligand and can dissociate to form a square-planar, cationic nickel complex with open coordination sites for transmetalation (39). Following transmetalation with lithium-salt-free organozinc reagents (40), reductive elimination from the five-coordinate (tpy) $\mathrm{Ni}^{\mathrm{II}} \mathrm{R}_{2}$ should be fast (41).

In developing synthetic applications of this ring-opening reaction, we found two sets of conditions that allowed the use of a variety of coupling partners while employing a single nickel catalyst (Fig. 4). The first is an auto-tandem process, operationally like a crosselectrophile coupling (22), where aryl halides are converted in situ into arylzinc reagents by the same nickel catalyst that performs the $\mathrm{C}-\mathrm{C}$ activation/cross-coupling (Fig. S14-S17). The second is a decoupled version, wherein an aryl or vinyl halide is converted to the corresponding organozinc halide in one flask by (tpy) $\mathrm{Ni}^{\mathrm{II}} \mathrm{Cl}_{2}$ and a zinc reductant, followed by immediate use of this reagent in the $\mathrm{C}-\mathrm{C}$ activation/cross-coupling in a second flask. Both sets of conditions provided the desired products as the TMS silyl enol ethers with $\geq 95: 5 \mathrm{Z} / E$ stereoselectivity.

The resulting reaction is robust, coupling a variety of aryl and alkyl cyclopropyl ketones with a diverse set of aryl, alkenyl, and alkylzinc reagents (Fig 4). Efficient 1,3difunctionalization of (hetero)arylzinc reagents occurred regardless of arene electronics ( $\mathbf{3 b}$, 3c, 3g, 3h), Lewis basic heteroatoms (3k, 3p), or reactive functionalities that can be used in subsequent cross-coupling reactions $(\mathbf{3 e}, \mathbf{3 j})$. More complex cyclopropyl ketones, including those with additional substitution and bicycles, were reactive, demonstrating that the reaction is not limited to aryl cyclopropyl ketones and can be further expanded to alkyl analogs (3ae3ah). An enantioenriched bicyclic cyclopropyl ketone substrate afforded the cross-coupled product with complete retention of the $\beta$-stereocenter $((\boldsymbol{R})-3 \mathbf{a e})$. While more hindered cyclopropyl ketones coupled in lower yield (3ai-3ak), improved reactivity was obtained by adjusting our ligand to be less sterically demanding but still easily reduced. Bidentate $4,4^{\prime}$ bis(carboxymethyl)-2,2'-bipyridine (L2) with added $N, N$-dimethylaminopyridine was effective for products 3ai, 3aj, and 3ak, suggesting that ligand-nickel cooperative mechanisms might be general for a variety of catalysts.

Besides arylzinc reagents, alkenyl and alkyl zinc reagents both worked with minimal adjustment to the reaction conditions and demonstrated functional-group compatibility consistent with other Negishi-type cross-coupling reactions. Notably, esters (3al), ethers (3am), protected amines (3aq), alkyl trimethoxysilane (3ao), and alkyl boronic acid pinacol esters (3an) were all compatible. Sterically hindered zinc reagents, such as ortho-substituted arylzincs and $2^{\circ}$ alkyl zinc reagents, coupled in low yield under these conditions.

Synthetically, this reaction is notable because there are few examples of $C\left(\mathrm{sp}^{3}\right)-C\left(\mathrm{sp}^{3}\right)$ bond activation/skeletal rearrangements with concomitant $\mathrm{C}\left(\mathrm{sp}^{3}\right)-\mathrm{C}\left(\mathrm{sp}^{3}\right)$ cross-coupling. When this reaction is paired with a second $\mathrm{C}\left(\mathrm{sp}^{3}\right)-\mathrm{C}\left(\mathrm{sp}^{3}\right)$ bond-forming reaction at the silyl enol ether (Fig. 1C), this process will facilitate the insertion of saturated triads between two other components. Even in this initial proof-of-concept iteration, and without exploiting the power of silyl enol ethers, this method can be used to shorten syntheses. For example, in Fishlock's ( \pm )-taiwaniaquinol B synthesis we were able to decrease the step count to intermediate 3au by three steps (out of 7) (42). Our four-step route utilized a different starting material (5) of similar complexity and the same oxidation state as in the Fishlock synthesis.

A key feature of this method is access to reliable difunctionalization (Fig. 1C) and this requires isolation of reactive silyl enol ethers. To maximize yield and simplify isolation, many reports either hydrolyze the enol ethers and isolate the ketone or substitute a more stable, but 
less useful silicon group for isolation. We found that careful control of $\mathrm{pH}$ in the workup coupled with non-aqueous reverse phase chromatography allowed for relatively easy isolation in yields identical to the GC yield ( $80 \% \mathrm{GC}$ yield, $80 \%$ isolated yield of 3a) (43). While once cost-prohibitive, the proliferation of automated chromatography instruments and reusable stationary phases has dramatically lowered the barrier to employing reverse-phase purification technologies on preparative scale.

In conclusion, these studies illustrate the potential of new mechanisms of $\mathrm{C}-\mathrm{C}$ activation in synthesis by showing how a novel metalloradical mechanism enables the first general $\mathrm{C}-\mathrm{C}$ activation of cyclopropyl ketones to enable 1,3-difunctionalization. This metalligand cooperativity is a new application of redox-active ligands that could be of wide use in catalysis. We anticipate that these results will enable the rapid development of a variety of $\mathrm{C}-$ $\mathrm{C}$ activation/difunctionalization reactions of cyclopropyl ketones. 
A Known: 3-center 2-electron oxidative $\mathrm{C}-\mathrm{C}$ activation of cyclopropanes

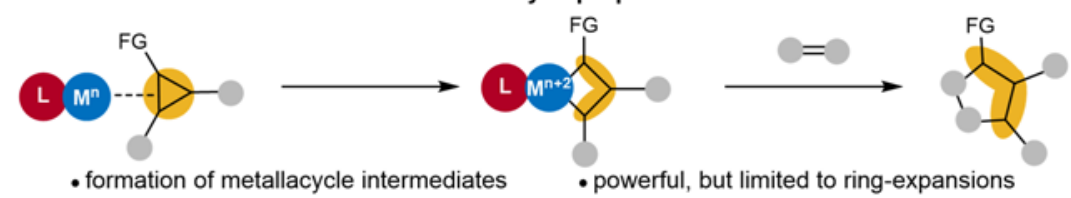

B New activation mode enables merging $\mathrm{C}-\mathrm{C}$ activation with cross-coupling

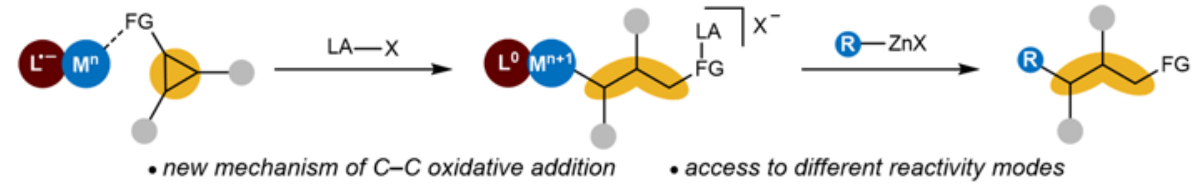

C Cyclopropyl ketones: new versatile synthons for 1,3-difunctionalization

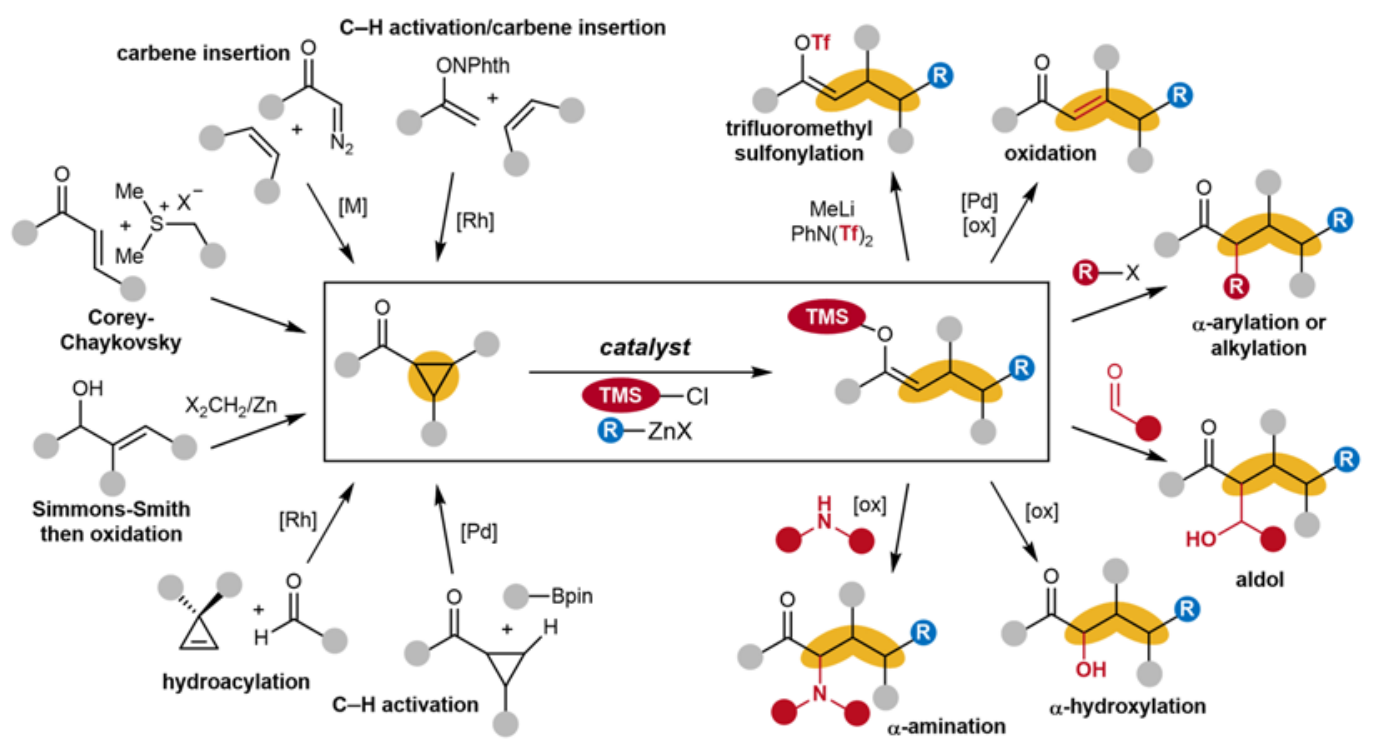

D This work: metal-ligand cooperativity enables $\mathrm{C}-\mathrm{C}$ activation/cross-coupling instead of ring expansion

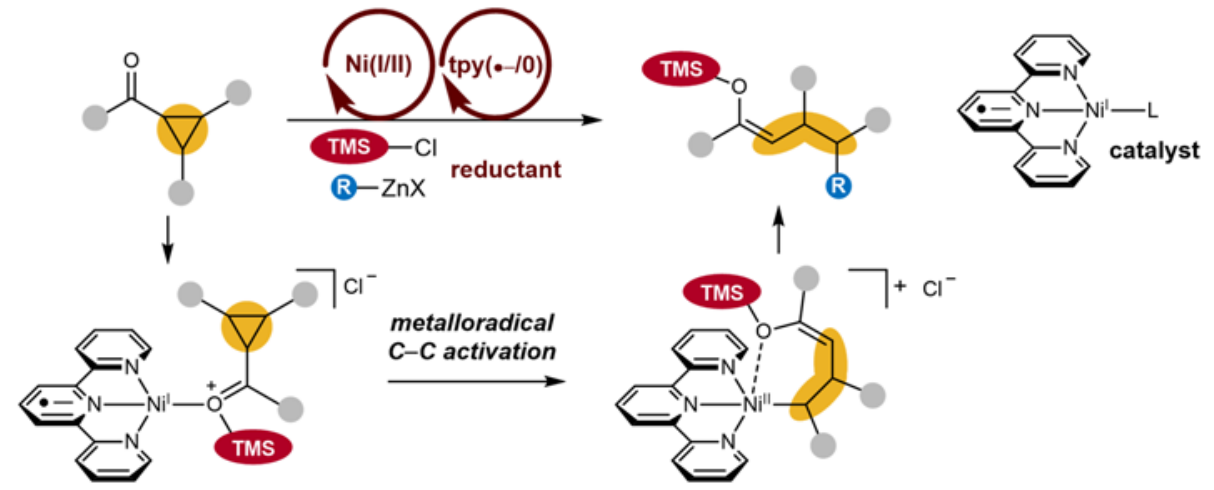

Fig. 1. Cyclopropyl ketone $\mathrm{C}-\mathrm{C}$ bond activation/cross-coupling. (A) Known: 3-center 2-electron oxidative $\mathrm{C}-\mathrm{C}$ activation of cyclopropanes. (B) Merging $\mathrm{C}-\mathrm{C}$ activation with cross-coupling. (C) Cyclopropyl ketones: new versatile synthons for 1,3-difunctionalization. (D) This work: metalligand cooperativity enables $\mathrm{C}-\mathrm{C}$ activation/cross-coupling instead of ring expansion. L, ligand; M, transition metal; FG, functional group; LA, Lewis acid; X, halide; Me, methyl; NPhth, 
phthalimide; TMS, trimethylsilyl; pin, pinacolato; R, aryl or alkyl substituent; Tf, trifluoromethanesulfonyl; Ph, phenyl; [ox], oxidant; tpy, terpyridine.

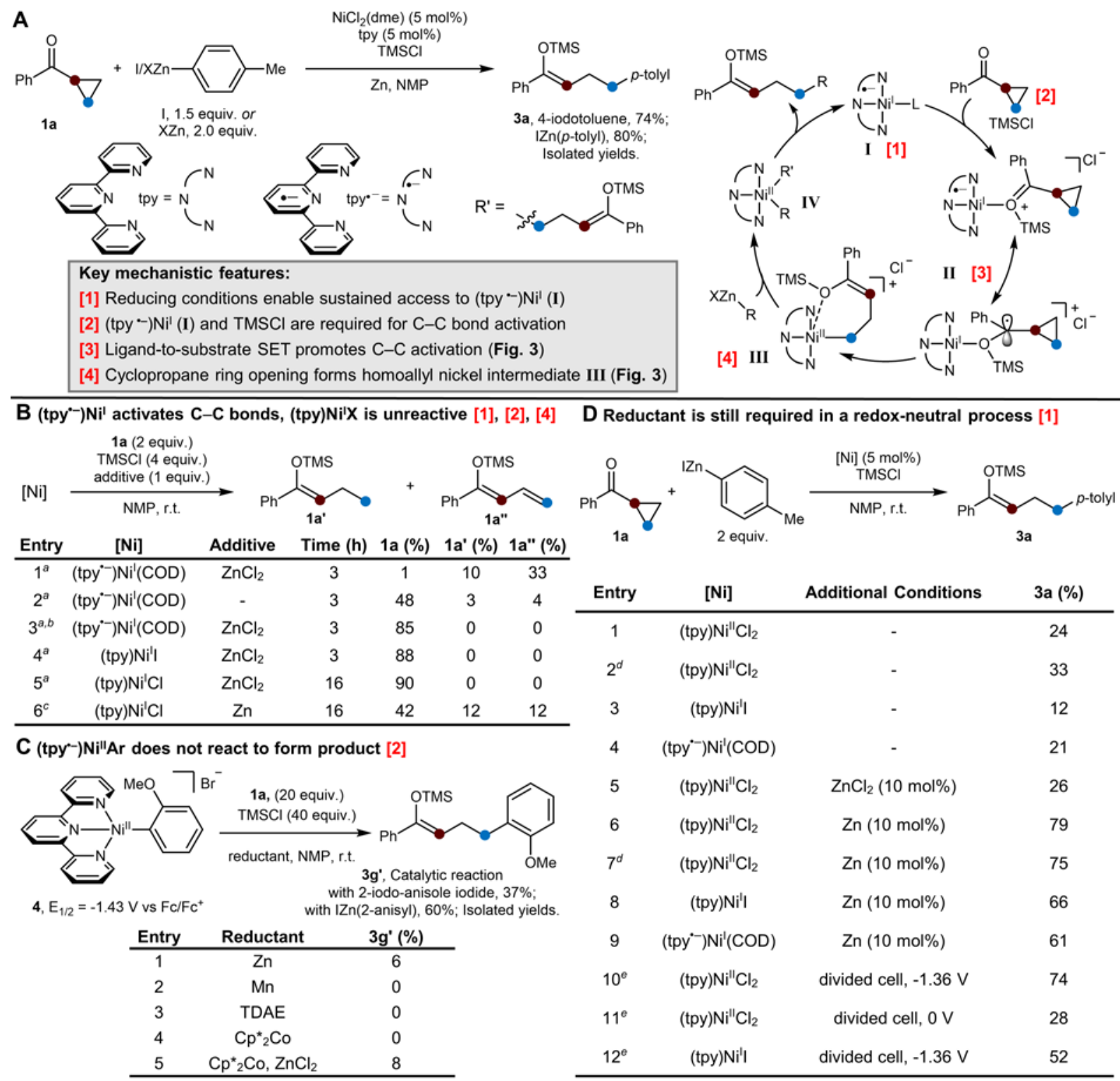

Fig. 2. Unified mechanistic proposal for cyclopropyl ketone $\mathrm{C}-\mathrm{C}$ activation / cross-coupling. (A) Optimized conditions and proposed mechanism. (B) I activates $\mathrm{C}-\mathrm{C}$ bonds, (tpy) $\mathrm{Ni}^{\mathrm{I} X}$ is unreactive. (C) $\left(\right.$ tpy $\left.^{*-}\right) \mathrm{Ni}^{\mathrm{II}}(\mathrm{Ar})$ is not competent for $\mathrm{C}-\mathrm{C}$ activation/cross-coupling. (D) Reductant is required to sustain catalytic activity. Unless otherwise stated, yields are calibrated GC yields vs. dodecane internal standard. ${ }^{a}$ Yields account for 1 equiv. excess 1a. ${ }^{b} \mathrm{TMSCl}$ omitted. ${ }^{c}$ Yields calculated based on the amount of $1 \mathbf{a}$ instead of [Ni]. ${ }^{d}$ Catalyst stirred over 2 equiv. activated $\mathrm{Zn}$ in NMP, then filtered prior to use. ${ }^{e}$ Voltage $\mathrm{vs}$. $\mathrm{Fc} / \mathrm{Fc}^{+}$, sacrificial $\mathrm{Zn}$ anode. dme, 
dimethoxyethane; COD, 1,5-cyclooctadiene; TDAE, tetrakis(dimethylamino)ethylene; $\mathrm{Cp}{ }_{2} \mathrm{Co}$, bis(pentamethylcyclopentadienyl)cobalt(II).

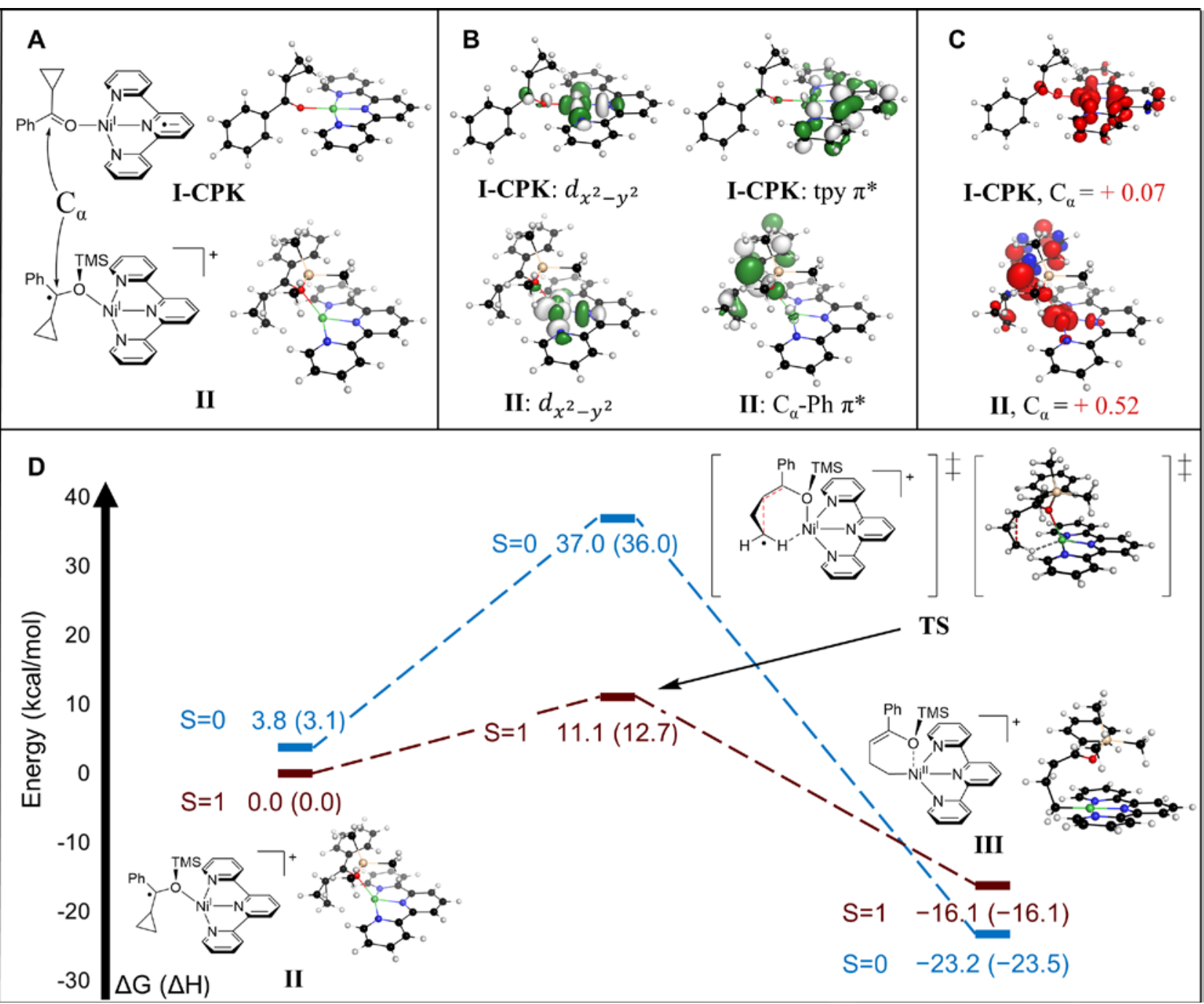

Fig. 3. DFT investigation into the Ni-mediated cyclopropane C-C activation. (A) Line drawings and DFT-calculated structures of I-CPK and II. (B) Singly-occupied molecular orbitals (SOMOs) consist of the $\mathrm{Ni} \mathrm{d}_{\mathrm{x} 2-\mathrm{y} 2}$ orbital and a $\pi^{*}$-symmetry orbital localized to either the terpyridine ligand (as in I) or the silyl carboxonium carbon $\left(\mathrm{C}_{\alpha}\right)$ of bound substrate (as in II). (C) Spin density plots reveal a significant transfer of electron density from the terpyridine ligand to bound substrate that is dependent on the presence of TMS cation. Based on molecular orbital occupancies and spin population analysis, I-CPK and II are best described as $\mathrm{Ni}^{\mathrm{I}}$ species with either a radical anion terpyridine ligand or a radical silyl carboxonium ligand, respectively. This difference in electronic structure is predicted to result in the different reactivity observed in the absence versus presence of TMSCl. (D) Calculated reaction coordinate for the transformation of II to Ni-alkyl intermediate III. The $\mathrm{C}-\mathrm{C}$ bond activation step is an asynchronous concerted process that avoids a free radical intermediate and exhibits spin crossover from $\mathrm{S}=1$ (II) to $\mathrm{S}=0$ (III). 
Submitted Manuscript: Confidential

Template revised February 2021

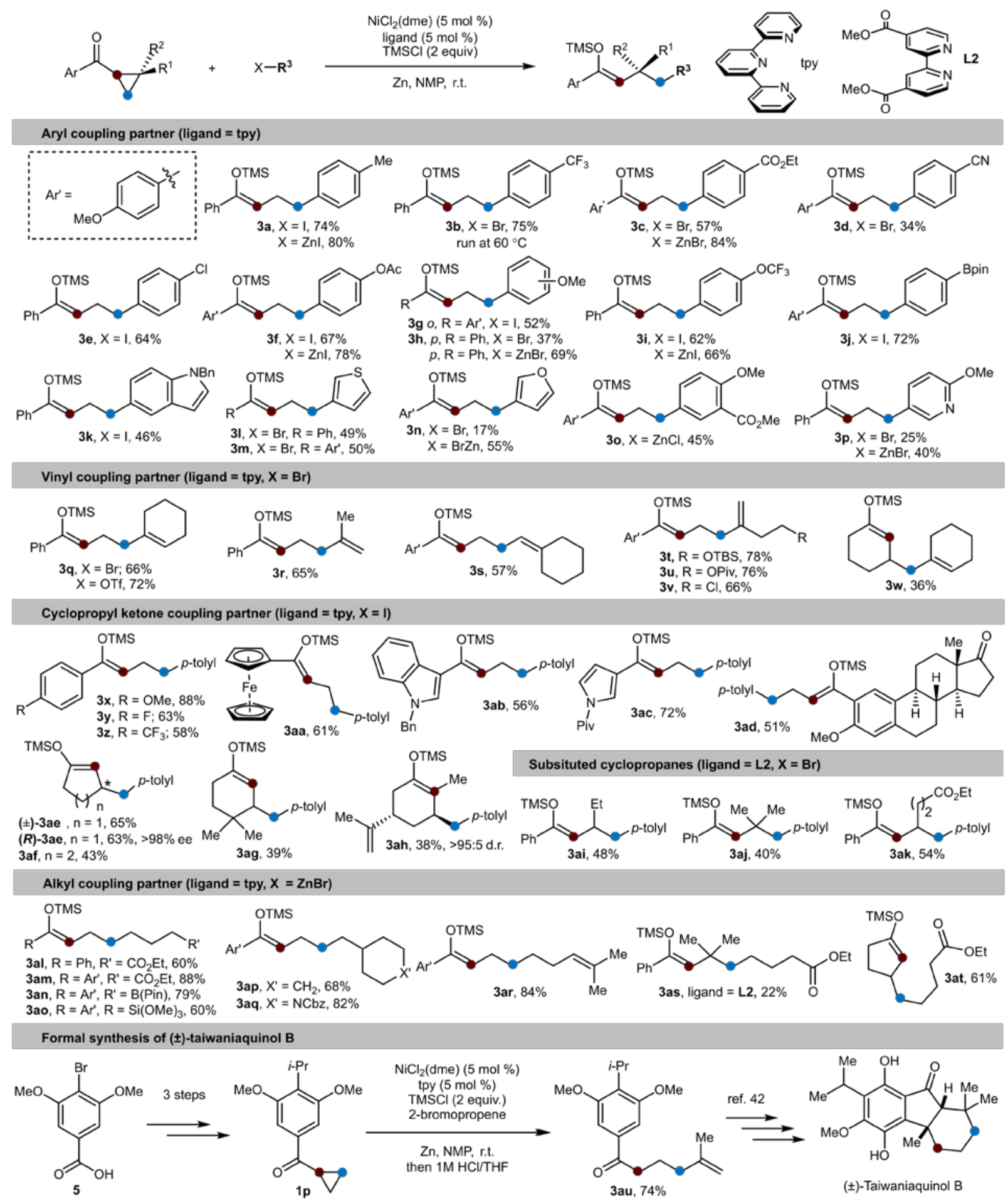

Fig. 4. Scope of the 1,3-difunctionalization reaction and the formal synthesis of ( \pm )-taiwaniaquinol B. Yields are isolated yields after purification. Et, ethyl; Ac, acetyl; Bn, benzyl; TBS, tertbutyldimethylsilyl; piv, pivaloyl; Cbz, benzyloxycarbonyl; $i$-Pr, isopropyl. 


\section{References and Notes}

1. B. Rybtchinski, D. Milstein, Metal Insertion into C-C Bonds in Solution. Angew. Chem. Int. Ed. 38, 870-883 (1999).

2. C.-H. Jun, Transition metal-catalyzed carbon-carbon bond activation. Chem. Soc. Rev. 33, 610-618 (2004).

3. J. J. Eisch, A. M. Piotrowski, K. I. Han, C. Kruger, Y. H. Tsay, Organic chemistry of subvalent transition complexes. 9. Oxidative addition of nickel $(0)$ complexes to carboncarbon bonds in biphenylene: formation of nickelole and 1,2-dinickelecin intermediates. Organometallics. 4, 224-231 (1985).

4. L. Souillart, N. Cramer, Catalytic C-C Bond Activations via Oxidative Addition to Transition Metals. Chem. Rev. 115, 9410-9464 (2015).

5. P.-H. Chen, B. A. Billett, T. Tsukamoto, G. Dong, "Cut and Sew" Transformations via Transition-Metal-Catalyzed Carbon-Carbon Bond Activation. ACS Catal. 7, 1340-1360 (2017).

6. L. Liu, J. Montgomery, Dimerization of Cyclopropyl Ketones and Crossed Reactions of Cyclopropyl Ketones with Enones as an Entry to Five-Membered Rings. J. Am. Chem. Soc. 128, 5348-5349 (2006).

7. T. Tamaki, M. Ohashi, S. Ogoshi, [3+2] Cycloaddition Reaction of Cyclopropyl Ketones with Alkynes Catalyzed by Nickel/Dimethylaluminum Chloride. Angew. Chem. Int. Ed. 50, 12067-12070 (2011).

8. S. Ogoshi, M. Nagata, H. Kurosawa, Formation of Nickeladihydropyran by Oxidative Addition of Cyclopropyl Ketone. Key Intermediate in Nickel-Catalyzed Cycloaddition. $J$. Am. Chem. Soc. 128, 5350-5351 (2006).

9. T. Tamaki, M. Nagata, M. Ohashi, S. Ogoshi, Synthesis and Reactivity of Six-Membered Oxa-Nickelacycles: A Ring-Opening Reaction of Cyclopropyl Ketones. Chem. Eur. J. 15, 10083-10091 (2009).

10. T. Ichiyanagi, S. Kuniyama, M. Shimizu, T. Fujisawa, Regioselective Ring-opening of Cyclopropyl Ketones with Organometallic Reagents. Chem. Lett., 1149-1150 (1997).

11. D. H. T. Phan, K. G. M. Kou, V. M. Dong, Enantioselective Desymmetrization of Cyclopropenes by Hydroacylation. J. Am. Chem. Soc. 132, 16354-16355 (2010).

12. J. R. Denton, H. M. L. Davies, Enantioselective Reactions of Donor/Acceptor Carbenoids Derived from $\alpha$-Aryl- $\alpha$-Diazoketones. Org. Lett. 11, 787-790 (2009).

13. D. Nam, V. Steck, R. J. Potenzino, R. Fasan, A Diverse Library of Chiral Cyclopropane Scaffolds via Chemoenzymatic Assembly and Diversification of Cyclopropyl Ketones. $J$. Am. Chem. Soc. 143, 2221-2231 (2021). 
14. E. J. T. Phipps, T. Rovis, Rh(III)-Catalyzed C-H Activation-Initiated Directed Cyclopropanation of Allylic Alcohols. J. Am. Chem. Soc. 141, 6807-6811 (2019).

15. T. Piou, T. Rovis, Rh(III)-Catalyzed Cyclopropanation Initiated by $\mathrm{C}-\mathrm{H}$ Activation: Ligand Development Enables a Diastereoselective [2+1] Annulation of N-Enoxyphthalimides and Alkenes. J. Am. Chem. Soc. 136, 11292-11295 (2014).

16. M. Wasa, K. M. Engle, D. W. Lin, E. J. Yoo, J.-Q. Yu, Pd(II)-Catalyzed Enantioselective C-H Activation of Cyclopropanes. J. Am. Chem. Soc. 133, 19598-19601 (2011).

17. Y. Cohen, A. Cohen, I. Marek, Creating Stereocenters within Acyclic Systems by C-C Bond Cleavage of Cyclopropanes. Chem. Rev. 121, 140-161 (2021).

18. W. Su, S. Raders, J. G. Verkade, X. Liao, J. F. Hartwig, Pd-Catalyzed $\alpha$-Arylation of Trimethylsilyl Enol Ethers with Aryl Bromides and Chlorides: A Synergistic Effect of Two Metal Fluorides as Additives. Angew. Chem. Int. Ed. 45, 5852-5855 (2006).

19. A. M. R. Smith, K. K. (Mimi) Hii, Transition Metal Catalyzed Enantioselective $\alpha-$ Heterofunctionalization of Carbonyl Compounds. Chem. Rev. 111, 1637-1656 (2011).

20. J. Matsuo, M. Murakami, The Mukaiyama Aldol Reaction: 40 Years of Continuous Development. Angew. Chem. Int. Ed. 52, 9109-9118 (2013).

21. G. D. Jones, J. L. Martin, C. McFarland, O. R. Allen, R. E. Hall, A. D. Haley, R. J. Brandon, T. Konovalova, P. J. Desrochers, P. Pulay, D. A. Vicic, Ligand Redox Effects in the Synthesis, Electronic Structure, and Reactivity of an Alkyl-Alkyl Cross-Coupling Catalyst. J. Am. Chem. Soc. 128, 13175-13183 (2006).

22. M. J. Goldfogel, L. Huang, D. J. Weix, in Nickel Catalysis in Organic Synthesis (John Wiley \& Sons, Ltd, 2020; http://onlinelibrary.wiley.com/doi/abs/10.1002/9783527813827.ch9), pp. 183-222.

23. D. Haas, J. M. Hammann, R. Greiner, P. Knochel, Recent Developments in Negishi CrossCoupling Reactions. ACS Catal. 6, 1540-1552 (2016).

24. V. B. Phapale, D. J. Cárdenas, Nickel-catalysed Negishi cross-coupling reactions: scope and mechanisms. Chem. Soc. Rev. 38, 1598-1607 (2009).

25. X. Hu, Nickel-catalyzed cross coupling of non-activated alkyl halides: a mechanistic perspective. Chem. Sci. 2, 1867-1886 (2011).

26. O. R. Luca, R. H. Crabtree, Redox-active ligands in catalysis. Chemical Society Reviews. 42, 1440-1459 (2013).

27. W. Hao, J. H. Harenberg, X. Wu, S. N. MacMillan, S. Lin, Diastereo- and Enantioselective Formal [3 + 2] Cycloaddition of Cyclopropyl Ketones and Alkenes via Ti-Catalyzed Radical Redox Relay. J. Am. Chem. Soc. 140, 3514-3517 (2018). 
28. J. T. Ciszewski, D. Y. Mikhaylov, K. V. Holin, M. K. Kadirov, Y. H. Budnikova, O. Sinyashin, D. A. Vicic, Redox Trends in Terpyridine Nickel Complexes. Inorg. Chem. 50, 8630-8635 (2011).

29. A. M. Tondreau, C. C. H. Atienza, J. M. Darmon, C. Milsmann, H. M. Hoyt, K. J. Weller, S. A. Nye, K. M. Lewis, J. Boyer, J. G. P. Delis, E. Lobkovsky, P. J. Chirik, Synthesis, Electronic Structure, and Alkene Hydrosilylation Activity of Terpyridine and Bis(imino)pyridine Iron Dialkyl Complexes. Organometallics. 31, 4886-4893 (2012).

30. M. Wang, J. England, T. Weyhermüller, K. Wieghardt, Electronic Structures of "LowValent" Neutral Complexes [NiL2]0 ( $\mathrm{S}=0 ; \mathrm{L}=$ bpy, phen, tpy) - An Experimental and DFT Computational Study. Eur. J. Inorg. Chem. 2015, 1511-1523 (2015).

31. A. Wuttig, J. S. Derrick, M. Loipersberger, A. Snider, M. Head-Gordon, C. J. Chang, F. D. Toste, Controlled Single-Electron Transfer via Metal-Ligand Cooperativity Drives Divergent Nickel-Electrocatalyzed Radical Pathways. J. Am. Chem. Soc. 143, 6990-7001 (2021).

32. A. P. Gingsberg, Magnetic exchange in transition metal complexes vi: Aspects of exchange coupling in magnetic cluster complexes. Inorganica Chimica Acta Reviews. 5, 45-68 (1971).

33. L. Hie, E. L. Baker, S. M. Anthony, J.-N. Desrosiers, C. Senanayake, N. K. Garg, NickelCatalyzed Esterification of Aliphatic Amides. Angew. Chem. Int. Ed. 55, 15129-15132 (2016).

34. K. R. Wolthers, N. S. Scrutton, Protein Interactions in the Human Methionine Synthase-Methionine Synthase Reductase Complex and Implications for the Mechanism of Enzyme Reactivation. Biochemistry. 46, 6696-6709 (2007).

35. J. R. Rumble, Ed., CRC Handbook of Chemistry and Physics (CRC Press/Taylor \& Francis, Boca Raton, FL, 99th Edition.).

36. V. V. Pavlishchuk, A. W. Addison, Conversion constants for redox potentials measured versus different reference electrodes in acetonitrile solutions at $25^{\circ} \mathrm{C}$. Inorg. Chim. Acta. 298, 97-102 (2000).

37. J. Fuchs, E. Irran, P. Hrobárik, H. F. T. Klare, M. Oestreich, Si-H Bond Activation with Bullock's Cationic Tungsten(II) Catalyst: $\mathrm{CO}$ as Cooperating Ligand. J. Am. Chem. Soc. 141, 18845-18850 (2019).

38. D. C. Nonhebel, The chemistry of cyclopropylmethyl and related radicals. Chem. Soc. Rev. 22, 347 (1993).

39. T. J. Anderson, D. A. Vicic, Direct Observation of Noninnocent Reactivity of $\mathrm{ZnBr} 2$ with Alkyl Halide Complexes of Nickel. Organometallics. 23, 623-625 (2004). 
40. A. Joshi-Pangu, M. Ganesh, M. R. Biscoe, Nickel-Catalyzed Negishi Cross-Coupling Reactions of Secondary Alkylzinc Halides and Aryl Iodides. Org. Lett. 13, 1218-1221 (2011).

41. M. T. Quirós, D. Collado-Sanz, E. Buñuel, D. J. Cárdenas, On the mechanism of the formation of alkyl-Ni( I ) catalysts. Chem. Commun. 57, 2424-2427 (2021).

42. E. Fillion, D. Fishlock, Total Synthesis of ( \pm -Taiwaniaquinol B via a Domino Intramolecular Friedel-Crafts Acylation/Carbonyl r-tert-Alkylation Reaction. J. Am. Chem. Soc. 127, 13144-13145 (2005).

43. H. J. C. F. Nells, A. P. De Leenheer, Isocratic Nonaqueous Reversed-Phase Liquid Chromatography of Carotenoids. Anal. Chem. 55, 270-275 (1983).

Acknowledgments: We thank Dr. Heike Hofstetter (UW-Madison CIC) for assistance with EPR experiments, Colleen Chernowsky and Prof. Zach Wickens (UW-Madison) for assistance with the divided cell experiments, and Prof. Shannon Stahl for helpful discussions. We thank Joe Barendt and Chiral Technologies for the kind donation of achiral SFC columns used in this work. Analytical data were obtained from the CENTC Elemental Analysis Facility at the University of Rochester, funded by NSF CHE-0650456. Instrumentation in the PBCIC was supported by NIH S10OD020022, NSF CHE-1048642; NIH S10 OK012245, and a generous gift from P. J. and M. M. Bender.

Funding:

NIH R01GM097243

DOE DE-SC0021021

NSF CHE-0650456

NIH S10OD020022

NSF CHE-1048642

NIH S10 OK012245

\section{Author contributions:}

Conceptualization: DJW, MMG

Methodology: MMG, DJW, VRL, MJT

Investigation: MMG, VRL, MJT

Visualization: MMG, DJW, MJT

Funding acquisition: DJW, JFB

Project administration: DJW

Supervision: DJW, JFB

Writing - original draft: MMG, DJW, MJT

Writing - review \& editing: MMG, VRL, MJT, JFB, DJW 
Competing interests: Authors declare that they have no competing interests.

Data and materials availability: "All data are available in the main text or the supplementary materials."

\section{Supplementary Materials}

$5 \quad$ Materials and Methods

Supplementary Text

Figs. S1 to S31

References (44-86) 